\title{
Pituitary Abscess: Review and Highlight of a Case Mimicking Pituitary Apoplexy
}

\author{
Godefroy Hardy St-Pierre, Sandrine de Ribaupierre, Brian W. Rotenberg, Carolyn Benson
}

Can J Neurol Sci. 2013; 40: 743-745

Initially described by Heslop in 1848 and Simmonds in 1914, there have since been multiple case reports in the literature of pituitary abscess, but only 3 series of 20 or more patients ${ }^{1-3}$ with this disorder, all within the last decade. Even with more then 250 cases presented so far, pituitary abscess remains a clinical enigma with many unknowns. There have been even fewer cases of pituitary abscess acutely symptomatic with pituitary apoplexy: notably, only one article links these two explicitly ${ }^{4}$.

\section{Case Report}

Our patient, a 49-year-old right handed male, presented with acute sudden onset holoencephalic headache. Additionally, he was feeling unwell, was nauseated with occasional vomiting and complained of dizziness. He had been previously suffering from chronic holoencephalic headaches for six months which were of insufficient severity for him to seek medical attention. He also described a change in his vision, mentioned as blurriness.
On examination, this patient was alert and oriented to name, time and location. His vision was $20 / 40$ on the right $(20 / 25$ pinhole) and 20/70 on the left (20/30 pinhole). His visual fields both on confrontation and automated fields showed bitemporal hemianopsia respecting the vertical midline. There was no papilledema on fundoscopy. The reminder of the cranial nerve exam was unremarkable. Endocrine workup revealed the patient to have central adrenal insufficiency, hypothyroidism and hypogonadism, and appropriate replacement therapy was initiated.

Further imaging was ordered and an MRI with gadolinium revealed abnormal cystic enhancement of the pituitary gland as well as thickening of the pituitary stalk. The cyst rim was isointense on $\mathrm{T} 1$ and $\mathrm{T} 2$ while its contents were slightly hypointense on $\mathrm{T} 1$ and hyperintense on T2. (Figure) A diffusion sequence was not performed. No compression of the optic chiasm was seen but inflammation and enhancement of the diaphragm sellae was observed. At that point in time, the patient
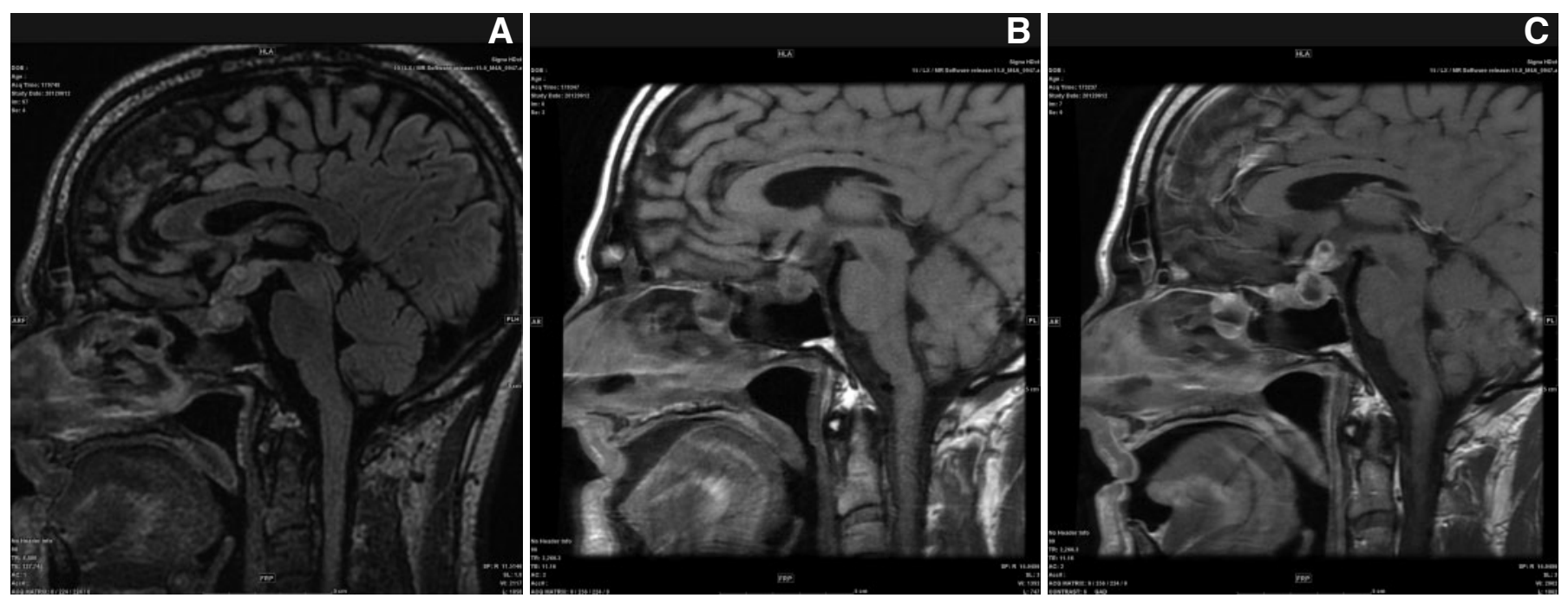

Figure: Pre-operative MRI (T1 Flair Pre \& Post; T2).

From the London Health Sciences Centre (GHS, SdR, CB), University Hospital; St. Joseph's Health Care (BWR), London, Ontario, Canada.

Received December 4, 2012. Final Revisions Submitted March 6, 2013.

Correspondence to: Godefroy Hardy St-Pierre, LHSC, University Hospital, 339 Windermere Road, London, Ontario, N6A 5A5, Canada. Email: ghardyst@uwo.ca. 
Table 1: Presenting symptom

\begin{tabular}{c|c|c|c|c}
\hline & Headache & Fever & $\begin{array}{c}\text { Visual } \\
\text { deficit }\end{array}$ & $\begin{array}{c}\text { Endocrine } \\
\text { deficit }\end{array}$ \\
\hline $\begin{array}{c}\text { Jain et al. } \\
(1997)\end{array}$ & 2 & 0 & 5 & 4 \\
\hline $\begin{array}{c}\text { Vates et al. } \\
(2001)\end{array}$ & 21 & 8 & 12 & 11 \\
\hline $\begin{array}{c}\text { Dutta et al. } \\
(2006)\end{array}$ & 4 & 4 & 2 & 4 \\
\hline $\begin{array}{c}\text { Liu et al. } \\
(2011)\end{array}$ & 21 & 4 & 9 & 27 \\
\hline $\begin{array}{c}\text { Zhang et al. } \\
(2012)\end{array}$ & 21 & 4 & 10 & - \\
\hline $\begin{array}{c}\text { Weighted } \\
\text { average } \%\end{array}$ & $\mathbf{7 4} \%$ & $\mathbf{1 9} \%$ & $\mathbf{4 1 \%}$ & $\mathbf{7 2} \%$ \\
\hline
\end{tabular}

significantly improved on medication but his visual fields remained unchanged. Decision was taken to bring him to the OR for a pituitary biopsy to confirm the diagnosis of hypophysitis.

An endonasal endoscopic transsphenoidal approach to the pituitary fossa was performed. Upon opening the dura of the sella, thick yellow pus came out under pressure. Cultures sent at the time were positive for Staphylococcus epidermidis. The patient was started on IV cefotriaxone and vancomycin. Our patient suffered from diabetes insipidus post operatively and required desmopressin (dDAVP) supplementation on discharge as well as continuation of steroids and levothyroxine. Post operative imaging showed resolution of the abscess. At initial six week follow-up, the patient was still in a state on panhypopituitarism but had regained full visual fields. He had no further headache, no cerebrospinal fluid (CSF) leak and was overall much improved.

\section{Discussion}

Pituitary abscess is a very rare entity. Described in several surgical series of pituitary disorders, the reported frequency is between $0.2-1 \%{ }^{1}$ with the three largest series reporting $0.7 \%, 1 \%$ and $0.4 \%$. A slight female predominance is seen in most series $1.3: 1^{2}$ further compounded by the $1.8: 1$ and $1.4: 1$ ratios reported in the two Chinese series ${ }^{1,3}$. The University of California San Fransisco (UCSF) series however reports a 1.7:1 male predominance $^{2}$. The age ranges from 12-71 year-old with a mean age of 40-years-old.

The vast majority (62\%) of patients have no predisposing factors. Among these cases, while pus is identified at surgery, cultures are usually sterile with only $10 \%$ identifying a microorganism. If a pre-existing local or remote infection (19\%) is known, $83 \%$ cultures will be positive: more commonly, this represents local extension of infection from the paranasal sinuses although cavernous sinus thrombophlebitis and infected CSF fistula are also reported. The other two main factors are a previous surgery $(17 \%)$ and the presence of a pituitary lesion $(11 \%)$

Presenting symptoms in pituitary abscesses are varied but usually entails headache and endocrine dysfunction. (Table 1). Diabetes insipidus was framed as a distinguishing factor
Table 2: Outcome

\begin{tabular}{c|c|c|c|c}
\hline & $\begin{array}{c}\text { Full } \\
\text { recovery }\end{array}$ & $\begin{array}{c}\text { Endocrine } \\
\text { deficit }\end{array}$ & Panhypopituitarism & Death \\
\hline $\begin{array}{c}\text { Jain et al. } \\
(1997)\end{array}$ & 2 & - & - & 0 \\
\hline $\begin{array}{c}\text { Vates et al. } \\
(2001)\end{array}$ & 9 & 11 & 7 & 2 \\
\hline $\begin{array}{c}\text { Dutta et al. } \\
(2006)\end{array}$ & - & 3 & - & 1 \\
\hline $\begin{array}{c}\text { Liu et al. } \\
(2011)\end{array}$ & 8 & 22 & - & 0 \\
$\begin{array}{c}\text { Zhang et al. } \\
(2012)\end{array}$ & - & - & - & $\mathbf{3} \%$ \\
\hline $\begin{array}{c}\text { Weighted } \\
\text { average\% }\end{array}$ & $\mathbf{3 2} \%$ & $\mathbf{6 2} \%$ & $\mathbf{2 9} \%$ & \\
\hline
\end{tabular}

suggesting pituitary abscess giving its relative rarity in the pituitary adenoma. Indeed, $56 \%$ of selected cases had diabetes insipidus but an even higher $68 \%$ had some form of anterior pituitary dysfunction.

Apoplexy in the setting of a pituitary abscess has been described once ${ }^{4}$. Contrary to our patient, that particular case presented with a decreased level of consciousness. Even then, the evacuation procedure took place six weeks after the initial presentation, further proof that pituitary abscess is a exceedingly difficult diagnosis to make.

Imaging appearance of pituitary abscess is deceptively non specific. Rim enhancement with gadolinium remains the most significant feature suggesting pituitary abscess. Those characteristics are unfortunately shared by the much more common necrotic pituitary adenoma as well as potentially evoking Rathke's cleft cyst or craniopharyngioma. The use of a diffusion-weighted sequence to refine the diagnosis appears promising, although its inclusion usually requires suspicion by the reporting neuroradiologist of a pituitary abscess.

Treatment of pituitary abscess entails mandatory surgical drainage. While transsphenoidal surgery is currently advocated as the surgical route by most case reports and series in the literature, cases of endonasal endoscopic transsphenoidal treatment of a pituitary abscess have been published in the literature $^{1}$. Improved visualization of the sellar content along with better appreciation of normal anatomy such as the cavernous sinus wall and the pituitary gland are brought forward as favouring endoscopy, but experience in regards to pituitary abscess remains minimal to date.

Outcomes are nowadays surprisingly good considering the original grim descriptions of the entity. (Table 2) All mortality associated with pituitary abscesses was reported in $1979^{2}$ or prior with the exception of two cases in $2006^{5}$ and $2008^{6}$. Compared to widely quoted mortality of $30-50 \%$, this represents a mortality of $3 \%$. Keeping in mind significant reporting bias, this much lower value is probably in keeping with the chronic indolent pathology often diagnosed post-operatively that constitutes most cases of pituitary abscesses in the modern literature. $^{3}$ 


\section{Conclusion}

Pituitary abscess is a rare clinical occurrence. Most cases are found incidentally and diagnosed intra-operatively when pus is delivered from the pituitary fossa. Initially reported as an often fatal entity, the recent literature rather suggests that it represent a chronic indolent process, explaining its frequent misdiagnosis as a pituitary adenoma. Acute presentation is unique and this report is, to our knowledge, the second pituitary abscess presenting with apoplexy ever published. Endocrine dysfunction is a common clinical presentation, a distinguishing feature helpful in differential diagnosis. Endonasal endoscopic transsphenoidal drainage, which offers optimal visualization of the sellar content, is considered to be the current best available treatment.

\section{REFERENCES}

1. Lui F, Li G, Yao Y, et al. Diagnosis and management of pituitary abscess: experience from 33 cases. Clin Endocrinol (Oxf). 2011; 74(1):79-88.

2. Vates GE, Berger MS, Wilson CB. Diagnosis and management of pituitary abscess: a review of 24 cases. J Neurosurg. 2001;95(2): 233-41.

3. Zhang X, Sun J, Shen M, et al. Diagnosis and minimally invasive surgery for the pituitary abscess: a review of 29 cases. Clin Neurol Neurosurg. 2012;114(7):957-61 .

4. Kindgon CC, Sidhu PS, Cohen J. Pituitary apoplexy secondary to an underlying abscess. J Infect. 1996;33(1):53-5.

5. Dutta P, Bhansali A, Singh P, Kotwal N, Pathak A, Kumar Y. Pituitary abscess: report of 4 cases and review of the literature. Pituitary. 2006;9(3):267-73.

6. Jain KC, Varma A, Mahapatra AK. Pituitary abscess: a series of 6 cases. Br J Neurosurg. 1997;11(2):139-43. 